\title{
Lavandula angustifolia Miller e sua utilização na Odontologia: uma breve revisão
}

\author{
Lavandula angustifolia Miller and its use in Dentistry: a brief review \\ Lavandula angustifolia Miller y su uso en la Odontología: una breve revisión \\ Elaine Roberta Leite de SOUZA ${ }^{1}$ \\ José Henrique de Araújo $\mathbf{C R U Z}^{1}$ \\ Nílvia Maria Lima GOMES \\ Laíse Luz RAMOS \\ Abrahão Alves de OLIVEIRA FILHO² \\ ${ }^{1}$ Graduandos em Odontologia da Universidade Federal de Campina Grande UFCG, Campus de Patos 58708-110, Patos-PB, Brasil \\ ${ }^{2}$ Professor Doutor do Curso de Odontologia da Universidade Federal de Campina Grande, UFCG, Campus de Patos 58708-110, Patos-PB, Brasil
}

\begin{abstract}
Resumo
O uso de medicamentos fitoterápicos vem crescendo mundialmente entre os programas preventivos e curativos, e tem estimulado a avaliação dos extratos de plantas para o uso na odontologia como controle do biofilme dental e outras desordens bucais. As plantas do gênero Lavandula, pertencem à família Lamiaceae, e têm sido utilizadas através dos anos para uma variedade de propósitos cosméticos e terapêuticos. Sua utilização na odontologia é, na grande maioria dos estudos, devido ao seu potencial ansiolítico. Entretanto, a Lavandula angustifólia demonstra outros potencias farmacológicos, como sua atividade antimicrobiana, antifúngica, anti-inflamatória e antinociceptiva. O presente estudo teve como objetivo integrar os conhecimentos já existentes sobre os aspectos das propriedades farmacológicas da Lavandula angustifolia Miller e sua aplicação na Odontologia. Trata-se de uma revisão bibliográfica no qual foi realizada uma seleção de artigos científicos a partir das bases de dados: Lilacs, MEDLINE, BVS e Scielo, além de monografias que atenderam aos requisitos do tema abordado, no período 2008 a 2018 com exceção de artigos clássicos que se apresentaram imprescindíveis ao presente estudo. Obteve-se um total de 1.532 artigos. Foram selecionados 38 artigos como amostra, que apresentaram a temática elencada para a pesquisa e que foram divididos por sessões: aspectos botânicos da planta; aspectos bioquímicos da planta; potencial antimicrobano; potencial anti-inflamatório; potencial ansiolítico e; potencial antinociceptivo. Pode-se concluir que a Lavandula angustifólia Miller apresenta-se como uma boa alternativa para utilização na odontologia. Entretanto, a falta de trabalhos que abordem sua utilidade na odontologia revela a necessidade de se intensificar as pesquisas sobre o assunto.
\end{abstract}

Descritores: Plantas Medicinais; Odontologia; Lavandula.

\section{Abstract}

The use of herbal medicines has been growing worldwide among preventive and curative programs and has stimulated the evaluation of plant extracts for use in dentistry as a control of dental biofilm and other oral disorders. Lavandula plants belong to the Lamiaceae family, and have been used over the years for a variety of cosmetic and therapeutic purposes. Its use in dentistry is, in the great majority of studies, due to its anxiolytic potential. However, Lavandula angustifolia shows other pharmacological potencies, such as its antimicrobial, antifungal, antiinflammatory and antinociceptive activity. The aim of the present study was to integrate existing knowledge on aspects of the pharmacological properties of Lavandula angustifolia Miller and its application in dentistry. It is a bibliographic review in which a selection of scientific articles was carried out from the databases Lilacs, MEDLINE, BVS and Scielo, as well as monographs that met the requirements of the topic addressed, from 2008 to 2018, with the exception of classical articles that were essential to the present study. A total of 1,532 articles were obtained. A total of 38 articles were selected as the sample, which presented the theme for the research and were divided by sessions: botanical aspects of the plant; biochemical aspects of the plant; antimicrobial potential; anti-inflammatory potential; anxiolytic potential and; antinociceptive potential. It can be concluded that Lavandula angustifolia Miller presents itself as a good alternative for use in dentistry. However, the lack of studies that address its usefulness in dentistry reveals the need to intensify research on the subject.

Descriptors: Plants, Medicinal; Dentistry; Lavandula.

\section{Resumen}

El uso de medicamentos fitoterápicos viene creciendo mundialmente entre los programas preventivos y curativos, y ha estimulado la evaluación de los extractos de plantas para el uso en la odontología como control del biopelículas dental y otros desórdenes bucales. Las plantas del género Lavandula, pertenecen a la familia Lamiaceae, y han sido utilizadas a través de los años para una variedad de propósitos cosméticos y terapéuticos. Su uso en la odontología es, en la gran mayoría de los estudios, debido a su potencial ansiolítico. Sin embargo, la Lavandula angustifolia demuestra otras potencias farmacológicas, como su actividad antimicrobiana, antifúngica, anti-inflamatoria y antinociceptiva. El presente estudio tuvo como objetivo integrar los conocimientos ya existentes sobre los aspectos de las propiedades farmacológicas de la Lavandula angustifolia Miller y su aplicación en la Odontología. Se trata de una revisión bibliográfica en la que se realizó una selección de artículos científicos a partir de las bases de datos: Lilacs, MEDLINE, BVS y Scielo, además de monografías que atendieron a los requisitos del tema abordado, en el período 2008 a 2018 con excepción de artículos clásicos que se presentaron imprescindibles para el presente estudio. Se obtuvo un total de 1.532 artículos. Se seleccionaron 38 artículos como muestra, que presentaron la temática elaborada para la investigación y que fueron divididos por sesiones: aspectos botánicos de la planta; aspectos bioquímicos de la planta; potencial antimicrobiano; potencial anti-inflamatorio; potencial ansiolítico y; potencial antinociceptivo. Se puede concluir que la Lavandula angustifolia Miller se presenta como una buena alternativa para su uso en la odontología. Sin embargo, la falta de trabajos que abordan su utilidad en la odontología revela la necesidad de intensificar las investigaciones sobre el tema.

Descriptores: Plantas Medicinales; Odontología; Lavandula.

\section{INTRODUÇÃO}

$\mathrm{O}$ uso de medicamentos fitoterápicos vem crescendo mundialmente entre os programas preventivos e curativos, e tem estimulado a avaliação dos extratos de plantas para o uso na odontologia como controle do biofilme dental e outras desordens bucais. Dessa forma, a odontologia é beneficiada pela riqueza em recursos naturais oferecidos pela flora brasileira, pois os produtos naturais estão cada vez mais presentes nos consultórios médicos e odontológicos, muito embora a fitoterapia ser pouco difundida fora do meio acadêmico ${ }^{1}$.

As plantas do gênero Lavandula, pertencem à família Lamiaceae, e têm sido utilizadas através dos anos para uma variedade de propósitos cosméticos e terapêuticos. Por possuir mais de 30 espécies e dezenas de subespécies, foi assim dividida em 4 categorias principais: L. angustifolia (L. officinalis), popularmente conhecida como Lavanda Inglesa; $L$. 
stoechas é, algumas vezes, chamada de Lavanda Francesa; L. latifolia e L. intermedia. Esses diversos tipos de Lavanda possuem propriedades etnobotânicas similares e grandes quantidades de constituintes químicos ${ }^{2,3}$.

A lavanda é uma planta caracterizada principalmente pelo seu aroma, é proveniente da região Mediterrânea, porém é amplamente cultivada na França, Itália e Espanha ${ }^{4}$. É popularmente conhecida por lavanda ou alfazema, é um arbusto ou subarbusto ereto e aromático com caules, na maioria das vezes, lenhosos, sendo o nome derivado do latim "lavare" cujo significado "lavar", refere-se ao uso destas plantas aromáticas em banhos .

As plantas de lavanda podem ser cultivadas para diversos fins. $\mathrm{Na}$ área comercial, estas plantas são muito utilizadas para a obtenção do óleo essencial, através da destilação de folhas e flores, sendo este produto bastante empregado na fabricação de fármacos, perfumes, cosméticos, entre outros; ou, ainda, na medicina popular devido às suas propriedades anestésicas, sedativas, antiespasmódicas, antibacteriana, entre outras ${ }^{5,6,7}$.

Sua utilização na odontologia é, na grande maioria dos estudos, devido ao seu potencial ansiolítico $^{8,9}$. Entretanto, a Lavandula angustifólia demonstra outros potencias farmacológicos além de seu potencial ansiolítico, como sua atividade antimicrobiana, antifúngica, anti-inflamatória e antinociceptiva. Diante do exposto, o objetivo do presente estudo é integrar os conhecimentos já existentes sobre os aspectos das propriedades farmacológicas da Lavandula angustifolia Miller e a aplicabilidade de tais atividades na Odontologia.

\section{MATERIAL E MÉTODO}

O presente estudo trata-se de uma revisão bibliográfica do tipo narrativa, no qual foi realizada uma seleção de artigos científicos recuperados a partir das bases de dados: Lilacs (Centro LatinoAmericano e do Caribe de Informação em Ciências da Saúde), MEDLINE (Medical Literature Analysis and Retrieval Sistem Online), BVS (Biblioteca Virtual em Saúde) e Scielo (Scientific Eletronic Library Online), além de monografias que atenderam aos requisitos do tema abordado, apresentando informações confiáveis e publicados em bases de dados no período 2008 a 2018 com exceção de artigos clássicos que se apresentaram imprescindíveis ao presente estudo.

A pesquisa foi realizada de 13 de janeiro a 24 de abril de 2018, nos idiomas português e inglês. A estratégia de busca utilizada para pesquisa nas bases de dados se deu pela utilização dos seguintes descritores isolados ou em combinação, nas linguagem português e inglês: "aspectos botânicos da planta "Lavandula angustifolia", "constituintes químicos da planta", "atividades farmacológicas da espécie", "aplicação na Odontologia", "lavanda", "alfazema", "fitoterapia", "antimicrobiano", "antiinflamatório", "antinociceptiva", "ansiolítica", "antifúngico", obtendo-se um total de 1.532 artigos. Foram selecionados 38 artigos como amostra, que apresentaram a temática elencada para a pesquisa e que foram discutidos nas seguintes sessões: a) aspectos botânicos da planta; b) aspectos bioquímicos da planta; c) potencial antimicrobiano; d) potencial anti-inflamatório; e) potencial ansiolítico e; f) potencial antinociceptivo.

\section{RESULTADOS E DISCUSSÃO}

- Aspectos botânicos da planta

A Lavandula angustifolia Miller pertence à família Lamiaceae, gênero Lavandula, conhecidas popularmente como lavanda-comum, lavandaverdadeira, alfazema ou lavanda-inglesa. Tal planta é nativa das regiões montanhosas do Mediterrâneo sendo cultivada principalmente na França, Itália e Espanha ${ }^{4,5}$.

É caracterizada como um subarbusto perene, apresentando uma altura de aproximadamente 60-70 $\mathrm{cm}$, odorífero, reto, e com ramificações extensas. Sua folhagem apresenta-se opostas, lineares e com bordas revolutas de coloração acinzentada, sendo que as folhas mais jovens se apresentam com uma cor mais sutil $^{5,6,10}$.

O tricoma das folhas, parte responsável pela secreção dos óleos essenciais, derivados do metabolismo secundário dessa planta, apresenta-se em tamanhos e formas diferentes, encontrando-se geralmente em ramos e conectadas as glândulas. As inflorescências possuem pedúnculo de tamanho variado, encontrando-se único ou ramificado, sendo os ramos laterais menores e a ramificação sob a linha da folhagem ${ }^{5,6,10}$

As flores apresentam cálice de $4-5 \mathrm{~mm}$ de comprimento com pequenos dentes e um apêndice suborbicular e podem se apresenta nas cores vermelha, roxa, banca ou verde sendo que a mais comum é sua apresentação na coloração azullilases ${ }^{5,6,10}$.

\section{- Aspectos bioquímicos da planta}

Vários estudos realizados ao longo dos anos têm demonstrado que a composição química da Lavandula angustifólia Miller sofre alterações de acordo com a parte da planta utilizada no estudo e os fatores ambientais ${ }^{4,11-13}$.

Duke $^{14} \mathrm{em}$ seu livro intitulado "Handbook of Medicinal Herbs" demonstrou que a Lavandula angustifolia tem em sua composição aproximadamente $0,5-1,5 \%$ de óleo essencial, flavonoides, taninos, cumarinas, e ácido ursólico, apresentando como principais componentes linalol (30-60\%), geraniol linalol e seus ésteres, lavandulol, nerol, 1-8 cineol, $\beta$-cariofileno, cumarina, limoneno, $\beta$-ocimeno, furfural,, tujona e pinocanfona. Outro 
estudo, realizado por Montovani ${ }^{15}$, encontrou também os terpenos epi- $\alpha$-muurolol e $\alpha$-bisabolol como compostos da Lavandula angustifólia (Quadro 1).

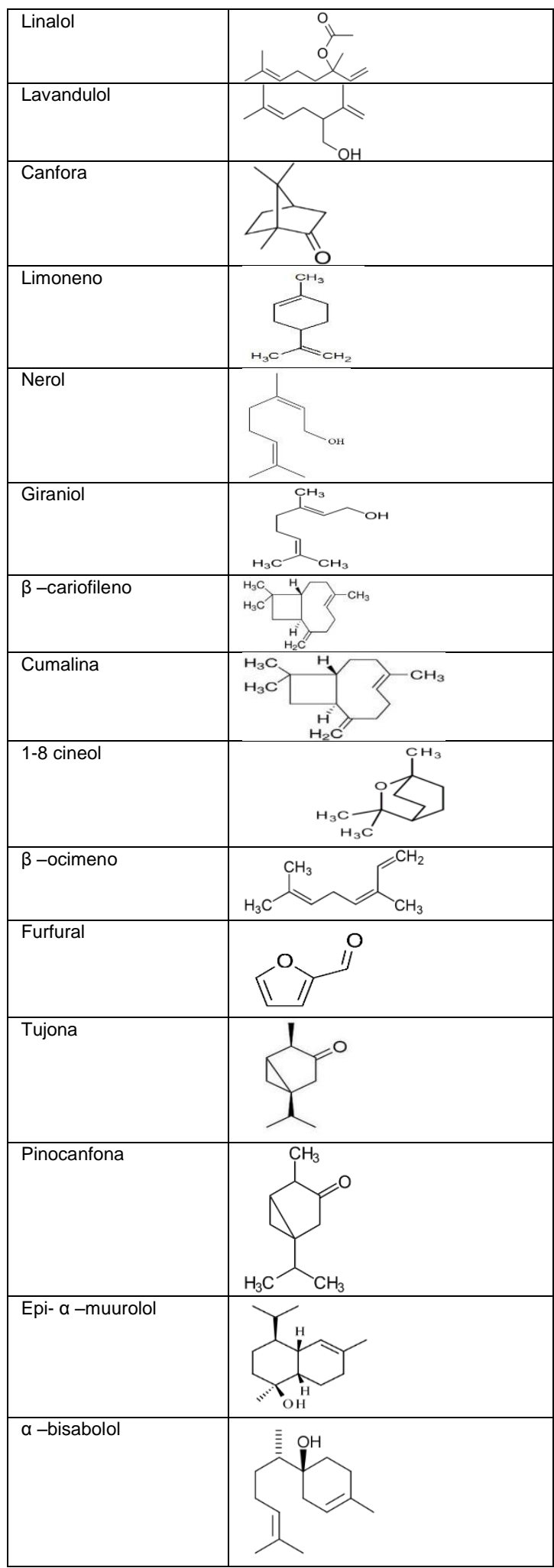

Quadro 1: Compostos encontrados na Lavandula angustifolia Miller

\section{- Potencial antimicrobiano}

O estudo realizado por Silveira et al. ${ }^{2}$ (2012) buscou determinar a composição química de óleos essenciais de Cymbopogon winterianus (DC) Stapf. (Poaceae) (citronela), Eucalyptus paniculata Sm. (eucalipto) e Lavandula angustifolia Miller. (Lamiaceae) (lavanda), e avaliar sua atividade antimicrobiana frente a 11 espécies de bactérias sendo 5 espécies Gram-positivas e 6 espécies Gram negativas: Staphylococcus monocytogenes, Enterococcus aureus, Listeria cereus, Bacillus faecalis , Bacillus coli , Salmonella typhimurium, Proteus vulgaris, Enterobacter aerogenes, Pseudomonas aeruginosa e Yersinia enterocolitica . Para a avaliação de seu potencial antimicrobiano foi realizado o método de disco-difusão e a determinação da Concentração Mínima Inibitória (CMI) e da Concentração Mínima Bactericida (CMB). Os resultados obtidos acerca da Lavandula angustifolia, de maior interesse na odontologia (potencial antimicrobiano contra Enterococcus faecalis, microrganismo presente em infecções dos canais radiculares) demonstraram que: (1) o óleo essencial de Lavandula angustifolia Miller apresentou o maior espectro de ação dentre os óleos essenciais avaliados, detectado no ensaio de difusão em disco, atividade antimicrobiana contra todos os micro-organismos testados, exceto P. aeruginosa, e atividade moderada contra E. faecalis; (2) Na determinação dos valores de CMI demonstrou boa atividade inibitória contra $\mathrm{E}$. faecali; (3) 1,8-cineol foi o principal constituinte identificado nesse óleo essencial (46,78\%).

Jianu et al. ${ }^{16}$ em seu trabalho, procurou investigar as propriedades antimicrobianas de óleos essenciais isolados de lavanda (L.angustifolia Miller) e de lavandim (Lavandula $x$ intermedia), assim como determinar as suas composições químicas. Os óleos foram isolados utilizando destilação a vapor por inflorescências, e posteriormente analisadas por cromatografia gasosa acoplada com espectrometria de massas(CG-EM). O método utilizado para avaliar a atividade antimicrobiana foi o Kirby-Bauer, no qual foi detectado ação bactericida dos dois óleos essenciais isolados sobre os microorganismos Shigella flexneri, Staphylococcus aureus, Escherichia coli e Salmonella typhimurium, enquanto que o Streptococcus pyogenes não se sensibilizou na presença de nenhum dos dois óleos essencias. Com isso, foi possível determinar que os óleos essencias da lavandula angustifolia $\mathrm{e}$ da lavandula $x$ intermedia se apresentaram com efetiva atividade bactericida mesmo isolados, na ausência do linalol ou do acetato de linalina, demonstrando que a ação antibacteriana das duas plantas medicinais é devida ao seus diferentes compostos químicos.

Outros microorganismos importantes são os fungos, como os pertencentes ao gênero Candida. A Candida albicans é um fungo oportunista naturalmente presente na cavidade oral mas que em determinadas condições, como a utilização de agentes imunossupressores, uso de antibióticos de amplo espectro, xerostomia, uso de próteses e má 
higienização oral pode torna-se patogênica aparecendo associada ou não a outras afecções ${ }^{17-19}$.

De acordo com Lam et al. ${ }^{17}$ as infecções causadas por fungos representam um desafio para a saúde pública e a crescente resistência a medicamentos antifúngicos exige o desenvolvimento de pesquisas científicas que busquem formas de tratamento alternativo e que sejam eficazes para o tratamento das lesões possibilitando a melhoria do bem-estar dos pacientes acometidos por esta doença.

$\mathrm{O}$ estudo de Rapper et al. ${ }^{20}$ teve como objetivo avaliar a interação in vitro das propriedades antimicrobianas de Lavandula angustifolia e uma seleção de óleos essenciais comumente usados em combinações terapêuticas. A eficácia antimicrobiana para o óleo essencial de Lavandula angustifolia foi investigado contra 14 patógenos, entre eles, a Candida albicans. Para isso, o ensaio de concentração mínima inibitória (CIM) de microdiluição foi realizado, pelo qual a fração inibitória de concentração ( $\Sigma$ FIC) foi calculada para as combinações dos óleos. Como resultados acerca de seu potencial para inibição da Candida, foi observado que a associação entre o óleo essencial de Lavandula angustifólia e Cupressus sempervirens ( $\Sigma$ FIC 0.15) e Lavandula angustifolia com Litsea cubeba ( $\mathrm{FFC}$ 0.19) apresentou o maior halo de inibição contra Candida albicans.

No entanto, estudos conduzidos por Uniyal et al. ${ }^{21}$ foi testada a eficácia de 16 tipos de óleos essenciais contra Aspergillus niger e A.fumigatus, espécies de fungos presentes na doença aspergilose formada por uma bola fúngica que se desenvolve no pulmão. Os vapores inalados dos óleos essencias funcionam matando os invasores nas paredes internas da via respiratória, e com isso contribuindo com o sistema imunológico do indivíduo afetado. Como resultado, 14 óleos essenciais se mostraram eficazes contra Aspergillus niger e A.fumigatus, e foram divididos em níveis de eficácia. Os mais eficazes foram os encontrados em Cinnamomum zeylanicum (Canela), Syzygium aromaticum (Cravo), Carum carvi (alcaravia), citratos de Cymbopogon (erva-cidreira), Foeniculum vulgare (funcho) e Myristica fragrans(Noz-moscada). Os de moderada eficácia foram encontrados em Gaultheria procumbens (Wintergreen), Pinus palustris (Terebintina), Sesamum indicum (Sesame), Trachyspermum ammi (Ajowain) e Origanum vulgare (Oregano). E os de mínima atividade foram encontrados na Lavandula angustifolia (Lavanda), Elletaria cardamomum (Cardamon) e Cymbopogon nardus (Citronella). E os dois tipos de óleos essenciais que não apresentaram nenhuma atividade foram da Azadirachta indica (Neem) e Linum usitatissimum (Linhaça).

\section{- Potencial anti-inflamatório}

$\mathrm{O}$ processo inflamatório pode ser definido como um mecanismo fisiopatológico de resposta do organismo à invasão por um agente infeccioso ou apenas uma reação a um dano tecidual de natureza química, térmica e mecânica, sendo representada por um conjunto de reações locais e gerais do organismo como a dor, rubor, calor, edema e perda de função ${ }^{19,22,23}$.

O estudo publicado por Silva ${ }^{24}$ avaliou o potencial antioxidante, anti-inflamatório e antinociceptivo do óleo essencial de lavanda (Lavandula angustifolia Mill) e o estudo da toxicidade aguda em camundongos. Para a verificação de sua toxicidade, os animais foram divididos em 5 grupos, escolhidos aleatoriamente: (1 - grupo controle) que foi tratado com água salina; (2) tratado com óleo essencial de lavanda na dose de $0,6 \mathrm{~g} / \mathrm{Kg}$; (3) tratado com óleo essencial de lavanda na dose de $1,5 \mathrm{~g} / \mathrm{kg}$; (4) tratado com óleo essencial de lavanda na dose de $3,0 \mathrm{~g} / \mathrm{Kg}$ e (5) tratado com óleo essencial de lavanda na dose de $5.0 \mathrm{~g} / \mathrm{Kg}$ e observados por 14 dias. Como resultado, obteve-se que o óleo essencial de lavanda na dose de $600 \mathrm{mg} / \mathrm{kg}$ é bem tolerado oralmente enquanto que doses acima de $1,5 \mathrm{~g} / \mathrm{Kg}$ demonstraram efeitos tóxicos.

Seu potencial anti-inflamatório foi testado através de dois modelos de inflamação aguda: pleurisia induzida por carragenina e edema de orelha induzido pelo óleo de cróton. Para a avaliação de sua atividade anti-inflamatória contra os danos causados pela carragenina, 3 grupos foram divididos aleatoriamente: (1) tratado com água salina; (2) tratados com óleo essencial de lavanda na dose de $600 \mathrm{mg} / \mathrm{Kg}$ e (3) tratados com Dexametasona na dose $500 \mathrm{mg} / \mathrm{Kg}$, uma hora antes da injeção intra-pleural da carragenina. Os resultados mostraram que a resposta edematogênica evocada por injeção intra-pleural de carragenina em ratos foi significativamente reduzida pelo pré-tratamento de animais com óleo essencial de lavanda; o óleo essencial causou uma redução acentuada de volume e proteína total concentração no exsudado recolhido; houve uma redução do total de células contagem e número de leucócitos polimorfonucleares que migraram para o espaço pleural da cavidade; e que a dexametasona, droga usada como referência, produziu efeitos semelhantes, mas significativamente mais eficaz que o óleo essencial. No entanto, apenas a lavanda óleo essencial reduziu significativamente a concentração de óxido nítrico no exsudado ${ }^{24}$.

Para a avaliação do potencial antiinflamatório tópico da lavanda o teste de edema de orelha foi utilizado. $\mathrm{Na}$ orelha direita, o óleo essencial de lavanda foi aplicado topicamente na concentração de $60 \mu \mathrm{l}$ enquanto em outro grupo aplicou-se dexametasona cerca de 1 hora antes do tratamento com óleo de cróton. Como resultados, viuse que a aplicação tópica o óleo essencial de Lavanda inibiu o desenvolvimento de edema de orelha e que esse efeito inibitório foi semelhante à inibição 
causada pela dexametasona ${ }^{24}$.

\section{- Potencial ansiolítico}

Cryan e Sweeney ${ }^{25}$ definem a ansiedade como sendo uma resposta fisiológica normal, que se manifesta em situações de perigo real ou imaginário. Tal resposta pode ser desencadeada por vários fatores que vão desde experiencia objetivas, subjetivas, o meio ambiente e até genéticas, podendo promover modificações nos sistemas neuroanatômico, neuroendócrino e dos neurotransmissores ${ }^{26-28}$.

A consulta odontológica por si só pode gerar ansiedade no paciente, ressaltados quando ocorre a necessidade de realização de procedimentos invasivos, como a aplicação de anestesia local ou cirurgias dentárias ${ }^{29}$. Outros estudos também revelam que devido a este medo, uma grande parte da população evita ir ao dentista, reconhecendo a ansiedade odontológica como uma das principais barreiras para as consultas clínicas ${ }^{30,31}$.

Um estudo realizado por Zabirunnisa et al. ${ }^{9}$ se propôs a avaliar efeito do óleo essencial de lavanda na ansiedade do paciente odontológico. A amostra contou com 597 pacientes de cinco clinicas odontológicas, que foram divididos em dois grupos: (1) odor ambiente de óleo essencial de lavanda, mantida com a ajuda de uma vela mais quente na zona de recepção e (2) grupo controle, vela mais quente com água normal. Os pacientes, após uma espera de 15 min em salas de espera condicionados, foram convidados a preencher o questionário com informações demográficas, como idade, sexo e a escala de ansiedade dental modificada, que é um breve questionário, auto-aplicado, consistindo em cinco perguntas em que sua somatória pode produzir uma pontuação total variando de 5 a 25 (ansiedade leve, moderada, grave). Os resultados mostraram que os pacientes submetidos ao cheiro da Lavanda, tiveram uma redução estatisticamente maior no nível de ansiedade que o grupo que não foi submetido.

No trabalho de Coelho et al..$^{32}$ procurou-se investigar os efeitos da inalação do óleo essencial da Lavandula angustifolia sobre a memória aversiva, assim como a sua influência na expressão do c-Fos, um gene envolvido em importantes eventos celulares como a hipóxia. Com isso, ratos Wistar machos adultos foram submetidos a um protocolo de condicionamento ao medo, e logo em seguida estes ratos permaneceram por durante 4 horas expostos a água vaporizada ou óleo essencial em soluções de $1 \%, 2,5 \%$ e $5 \%$. No dia seguinte, os ratos foram submetidos a testes de medo contextual ou tom, e após se passar 90 minutos foram sacrificados e submetidos a um processamento para observação dos seus cérebros na imunohistoquímica c-Fos. O óleo essencial a $2,5 \%$ e $5 \%$, e exceto o de $1 \%$, reduziu a resposta de congelamento e a expressão do c-Fos no hipocampo ventral e na amígdala com base no teste contextual. Já no teste de tom e medo, os ratos que inalaram o óleo essencial a 2,5\% e 5\% apresentaram redução do congelamento no três primeiros minutos após aplicação do tom, assim como reduziram a expressão de c-Fos no córtex pré-frontal e amígdala. Com isso, conseguiu-se demonstrar que os óleo essencial da Lavandula angustifolia apresentou efeito ansiolítico em animais condicionados a ansiedade.

\section{- Potencial antinociceptivo}

Segundo a Associação Internacional para o Estudo da Dor (IASP) a dor pode ser conceituada como uma experiência sensorial e emocional desagradável, relacionada à lesão tecidual real ou potencial, ou descritas em termos desse tipo de dano. Tal manifestação fisiológica pode ser classificada de acordo com o tipo de lesão e com os mediadores envolvidos, podendo ser de origem: neurogênica, quando há lesão do tecido neuronal; neuropática, quando há disfunção de um nervo; psicogênica, acarretada por fatores psicológicos; ou nociceptiva, quando há estimulação excessiva dos nociceptores ${ }^{33-35}$.

Assim, a crescente busca por métodos para alivio da dor, como a utilização de opioides e analgésicos tem gerado quadros de dependência e até morte. Dados publicados recentemente pelo Centers for Disease Control ${ }^{36}$ revelaram que nos Estados Unidos, o número de mortes por overdose de analgésicos triplicou de 1990 a 2008. Tal realidade motiva a pesquisa para métodos naturais para controle da dor que possibilitem sua diminuição e que tragam menos riscos à saúde da população.

Um estudo publicado em 2017 investigou o efeito da inalação do óleo essencial de Lavandula angustifolia Miller sobre a hiperalgesia mecânica em dois modelos animais de dor crônica e o possível envolvimento do sistema opioide neste efeito. Para isso, utilizou-se camundongos Swiss fêmeas submetidos à isquemia/reperfusão para indução da síndrome da dor regional complexa do tipo 1 (SDRC1) e a dor inflamatória crônica foi induzida pela injeção intraplantar (i.pl.) do adjuvante completo de Freund (CFA) e divididos em 4 grupos: grupo Naive $(\mathrm{N})$, composto por camundongos sem manipulação (CFA ou IR) e apenas avaliado; Grupo controle, composto por camundongos que foram submetidos a IR da pata, ou injeção de CFA e que inalaram salina; Grupo controle positivo, composto por camundongos que foram submetidos a IR da pata e tratados com gabapentina (30 mg/kg, i.g.) ou DC (10mg/kg, i.g.); $\mathrm{E}$ grupos experimentais, compostos por camundongos que foram submetidos a IR da pata injeção de CFA e tratados com inalação do óleo essencial de Lavandula angustifólia Miller em diferentes volumes $(100,200$ e $300 \mu \mathrm{l})$ por 30 minutos. Como resultados, foi observado que o grupo que recebeu a inalação do óleo essencial de Lavandula angustifolia Miller apresentou feito antihiperalgésico na SDRC-I e na dor inflamatória 
induzida por CFA (volume de $100 \mu \mathrm{l}$ ) e que o óleo essencial participou da ativação de receptores opioide periféricos e espinais ${ }^{37}$.

Em seu estudo, Maior ${ }^{38}$ investigou as propriedades farmacológicas do óxido de linalol (OXL), um monoterpeno, que pode ser obtido da oxidação natural ou por processos sintéticos do linalol, um composto presente da Lavandula angustifolia. Com isso foi avaliado as atividades ansiolíticas e antinociceptivas do OXL em camundongos Swins machos nos quais foram aplicados OXL pela via intraperitoneal (i.p.) nas doses de 50, 100 e $150 \mathrm{mg} / \mathrm{kg}$ e pela via inalatória (v.i.) nas concentrações de $0,65 \%, 1,25 \%, 2,5 \%, 5 \%$. Posteriormente, houve a realização de testes de triagem farmacológica comportamental, e o teste da barra giratória para observar a ação do OXL no sistema nervoso central. $\mathrm{O}$ efeito analgésico apresentou-se como o principal efeito observado nos camundongos tratados com OXL i.p. e v.i. durante o teste de triagem, e também observou-se que o mesmo não alterou a capacidade motora dos animas durante o teste da barra giratória.Com a realização de testes específicos foi observado o perfil farmacológico do OXL nas diferentes vias. O OXL via inalatória demonstrou perfil de droga ansiolítica por induzir os animais a permanecerem mais tempo nos braços abertos do labirinto e no compartimento iluminado do aparelho. Já nas contorções abdominais induzidas pelo ácido acético, o OXL via intraperitoneal apresentou a efetividade de uma droga antinociceptiva por diminui-las e pelo o tempo de lambida da pata dos animais observado no teste da formalina.

\section{CONCLUSÃO}

Diante disso, pode-se concluir que a Lavandula angustifólia Miller apresenta-se como uma boa alternativa para utilização na odontologia por apresentar vários potenciais farmacológicos vistos em diversos estudos. Entretanto, a falta de trabalhos que abordem sua utilidade na odontologia revela a necessidade de se intensificar as pesquisas sobre o assunto.

\section{REFERÊNCIAS}

1. Francisco KSF. Fitoterapia: uma opção para o tratamento odontológico. Rev Saúde. 2010; 4(1):18-24.

2. Silveira SM, Cunha Júnior A, Scheuermann GN, Secchi FL, Silvani V, Marisete $\mathrm{K}$ et al . Composição química e atividade antibacteriana dos óleos essenciais de Cymbopogon winterianus (citronela), Eucalyptus paniculata (eucalipto) e Lavandula angustifolia (lavanda). Rev Inst Adolfo Lutz. 2012;71(3):462-70.

3. Koulivand PH, Ghadiri MK, Gorji A. Lavender and the nervous system. Evid Based
Complement Alternat Med. 2013;2013:681304.

4. Verma RS, Rahman LU, Chanotiya CS, Verma RK, Chauhan A, Yadav A et al. Essential oil composition of Lavandula angustifolia Mill. cultivated in the mid hills of Uttarakhand, India. J Serb Chem Soc. 2010;75(3):343-48.

5. Biasi LA, Deschamps C. Plantas Aromáticas: do cultivo à produção de óleo essencial. Curitiba: Layer; 2009.

6. Lorenzi, H, Matos EJA. Plantas medicinais do Brasil: nativas e exóticas. 2. ed. Nova Odessa: Instituto Plantarum de Estudos da Flora; 2008.

7. Índice Terapêutico Fitoterápico (ITF). Petrópolis: Editora de Publicações Biomédicas; 2008.

8. Chioca LR. Avaliação do mecanismo de ação do efeito tipo ansiolítico da inalação do óleo essencial de lavanda em camundongos [tese]. Curitiba: Universidade Federal do Paraná; 2013.

9. Zabirunnisa M, Gadagi JS, Gadde P, Myla N, Koneru J, Thatimatla C. Dental patient anxiety: Possible deal with Lavender fragrance. J Res Pharm Pract. 2014;3(3):100-3.

10.Platt ES. Lavender: How to grow and use the fragrant herb. 2. ed. Mechanicsburg PA: Stackpole Books; 2009.

11.Hajhashemi V, Ghannadi A, Sharif B. Antiinflammatory and analgesic properties of the leaf extracts and essential oil of Lavandula angustifolia Mill. J Ethnopharmacol. 2003; 89(1):67-71.

12.Machado MP, Ciotta MN, Deschamps C, Zanette F, Côcco LC, Biasi LA. In vitro propagation and chemical characterization of the essential oil of Lavandula angustifolia cultivated in Southern Brazil. Cienc Rural 2013;43:283-89.

13.Porto C da, Decorti D, Kikic I. Flavour compounds of Lavandula angustifolia $\mathrm{L}$. to use in food manufacturing: Comparison of three different extraction methods. Food Chem. 2009; 112(4):1072-78.

14.Duke JA. Handbook of Medicinal Herbs. Flórida: CRC; 2000.

15.Mantovani ALL, Vieira GPG, Cunha WR, Groppo M, Santos RA, Rodrigues V et al . Chemical composition, antischistosomal and cytotoxic effects of the essential oil of Lavandula angustifolia grown in Southeastern Brazil. Rev. bras. farmacogn. 2013; 23(6):877-84.

16.Jianu C, Pop G, Gruia AT, Horhat FG. Chemical Composition and Antimicrobial Activity of Essential Oils of Lavender (Lavandula angustifolia) and Lavandin (Lavandula $\mathrm{x}$ intermedia) Grown in Western Romania. Int $\mathbf{J}$ Agric Biol. 2013;15(4):772-76.

17.Lam M, Jou Pc, Lattif Aa, Lee Y, Malbasa Cl, Mukherjee Pk et al. Photodynamic therapy with Pc 4 induces apoptosis of Candida albicans. Photochem Photobiol. 2011;87(4):904-9. 
18.Pereira CA, Romeiro RL, Costa AC, Machado AK, Junqueira JC, Jorge AO. Susceptibility of Candida albicans, staphylococcus aureus, and streptococcus mutans biofilms to photodynamic inactivation: an in vitro study. Lasers Med Sci. 2011;26(3):341-48.

19. Neville BW, Damm DD, Allen CM, Bouquot JE. Patologia Oral e Maxilofacial. 3. ed. Rio De Janeiro: Elsevier; 2009.

20.de Rapper S, Kamatou G, Viljoen A, van Vuuren S. The in vitro antimicrobial activity of lavandula angustifolia essential oil in combination with other aroma-therapeutic oils. Evid Based Complement Alternat Med. 2013;2013:852049.

21.Uniyal V, Bhatt RP, Saxena S, Talwar A. Antifungal activity of essential oils and their volatile constituents against respiratory tract pathogens causing Aspergilloma and Aspergillosis by gaseous contact. J Appl Nat Sci. 2012; 4(1):65-70.

22.Ribeiro BP, Pereira WS, Sousa AIP, Guerra RNM, Nascimento FRF. Alteração no perfil bioquímico induzido por reação inflamatória granulomatosa em camundondos. Rev Ciênc Saúde. 2010;12(1):73-9.

23.Rubin E, Gorstein F, Rubin R, Schwarting R, Strayer D. Patologia. Bases clínicopatológicas da Medicina. 4. ed. Rio de Janeiro: Guanabara Koogan; 2006.

24.Silva GL. Avaliação da atividade antioxidante, antiinflamatória e antinociceptiva do óleo essencial de lavanda (Lavandula angustifolia Mill) [dissertação]. Dissertação (Mestrado). Porto Alegre: Universidade Católica do Rio Grande do Sul; 2009.

25.Cryan, JF, Sweeney FF. The age of anxiety: role of animal models of anxiolytic action in drug discovery. Br J Pharmacol. 2011;164(4):1129-61.

26.Barik J, Marti F, Morel C, Fernandez SP, Lanteri $\mathrm{C}$, Godeheu $\mathrm{G}$ et al. Chronic stress triggers social aversion via glucocorticoid receptor in dopaminoceptive neurons. Science. 2013; 339(6117):332-35.

27. Martin EI, Ressler KJ, Binder E, Nemeroff CB. The neurobiology of anxiety disorders: brain imaging, genetics, and psychoneuroendocrinology. Psychiatr Clin North Am. 2009;32(3):549-75.

28. Oler JA, Fox AS, Shelton SE, Rogers J, Dyer TD, Davidson RJ et al. Amygdalar and hippocampal substrates of anxious temperament differ in their heritability. Nature. 2010;466(7308):864-68.

29.Loggia ML, Schweinhardt P, Villemure MC, Bushnell Mc. Effects of psychological state on pain perception in the dental environment. J Can Dent Assoc. 2008;74(7):651-56.

30.Carvalho RWF, Falcão PGCB, Campos GJL, Bastos AS, Pereira JC, Pereira MAS et al.
Ansiedade frente ao tratamento odontológico: prevalência e fatores predictores em brasileiros. Ciênc Saúde Colet. 2012;17(7):1915-22.

31.Siviero M, Nhani VT, Prado EFGB. Análise da ansiedade como fator preditor de dor aguda em pacientes submetidos à exodontias ambulatoriais. Rev Odontol UNESP. 2008;37(4):329-36

32. Coelho LS, Correa-Netto NF, Masukawa MY, Lima AC, Maluf S, Linardi A et al. Inhaled Lavandula angustifolia essential oil inhibits consolidation of contextual-but not tone-fear conditioning in rats. J Ethnopharmacol. 2018; 215:34-41.

33. Oliveira RRB, Góis RMO, Siqueira RS, Almeida JRGS, Lima JT, Nunes XP et al . Antinociceptive effect of the ethanolic extract of Amburana cearensis (Allemão) A.C. Sm., Fabaceae, in rodents. Rev bras farmacogn. 2009;19(3):672-76.

34.Millan MJ. Descending control of pain. Prog Neurobiol. 2002;66(6):355-474.

35.Julius D, Basbaum AI. Molecular mechanisms of nociception. Nature. 2001;413(6852):203-10.

36.Centers for Disease Control and Prevention. Unintentional drug poisoning in the United States. CDC: Atlanta, 2010. Disponível em: https://www.cdc.gov/homeandrecreationalsafety/p df/poison-issue-brief.pdf.

37.Donatello NN. Ativação de receptores opioides periféricos e espinais pela inalação do óleo essencial de lavandula augustifolia reduz hiperalgesia mecânica em modelos animais de neuropatia e inflamação crônica [dissertação]. Palhoça: Universidade do Sul de Santa Catarina, Pós-graduação em Ciências da Saúde; 2017.

38. Maior FNS. Atividade ansiolítica e antinociceptiva do óxido de linalol em modelos animais [tese]. João Pessoa: Universidade Federal da Paraíba; 2011.

\section{CONFLITO DE INTERESSES}

Os autores declaram não haver conflitos de interesse.

\section{AUTOR PARA CORRESPONDENCIA}

\section{Abrahão Alves de Oliveira Filho}

abrahao.farm@gmail.com

Submetido em 29/05/2018

Aceito em 27/06/2018 